\title{
CIUdADANÍA Y COMPETENCIA DIGITAL: SOBRE LOS PRESUPUESTOS DE UNA CIVILIZACIÓN DIGITAL GLOBAL A PARTIR DE LA INICIATIVA EUROPA 2020
}

\author{
CITIZENSHIP AND DIGITAL COMPETENCE; ON THE ASSUMPTIONS OF A GLOBAL \\ DIGITAL CIVILIZATION FROM THE INITIATIVE EUROPE 2020
}

\author{
Miguel MANDUJANO ESTRADA \\ Universidad de Barcelona \\ mmandujanoe@gmail.com
}

\author{
RECIBIDO: $21 / 07 / 2017$ \\ ACEPTADO: 03/11/2017
}

\begin{abstract}
Resumen: Valoraremos, en este trabajo, el discurso europeo de la ciudadanía digital a través del análisis de la iniciativa Europa 2020, llevado a cabo al inicio de la década para dar respuesta a los retos de la crisis. Criticaremos el modelo de civilización que supone el programa europeo, basado en el desarrollo e intercambio económico digital y el sentido estricto de alfabetización que comprende, y propondremos, en cambio, un significado más amplio que recorre un camino desde la noción de competencia hacia el de educación digital como una forma integradora y una premisa más justa para una civilización digital global.
\end{abstract}

Palabras clave: ciudadanía digital, competencia digital, educación digital, civilización digital global.

\begin{abstract}
In this work, we will assess the European discourse of digital citizenship through the analysis of the Europe 2020 initiative, carried out at the beginning of the decade to respond to the challenges of the crisis. We will criticize the European model of civilization, based on the development and digital economic exchange and the strict sense of literacy that it comprises, and we will instead propose a broader meaning that goes from the notion of competence to that of digital education, as an integrative form and a more just premise for a global digital civilization.
\end{abstract}

Keywords: digital citizenship, digital competence, digital education, global digital civilization.

\section{La iniciativa Europa 2020}

La idea de dedicar este trabajo a los presupuestos de una civilización digital global, y en particular, a la relación entre ciudadanía y competencia digital, comienza con una valoración de la iniciativa de la Comisión Europea Europa 2020 realizada en el cenit de la década para la que fue elaborada. Con este programa, la Comisión Europea calculaba hacer frente a las consecuencias negativas de la crisis sobre la base de tres prioridades: un crecimiento inteligente, un crecimiento sostenible y un crecimiento integrador. Indicaba, además, la premisa fundamental que debía cumplirse para alcanzar su meta: un marco más fuerte de gobernanza económica (Comisión Europea 2010, 6). 


\section{EUROPA 2020: OBJETIVOS PRINCIPALES}

- La tasa de empleo (población entre 20 y 64 años) debería pasar de $69 \%$ a 75\%.

- Alcanzar el objetivo de invertir el $3 \%$ del PIB en I+D, mejorando las condiciones por parte del sector privado y desarrollando un nuevo indicador que haga un seguimiento de la innovación.

- Reducir las emisiones de gases de efecto invernadero en un $20 \%$ en comparación con los niveles de 1990, incrementar el porcentaje de las energías renovables en nuestro consumo final de energía al 20\% y aumentar un $20 \%$ la eficacia en el uso de la energía.

- Reducir el porcentaje de abandono escolar del 15\% al 10\% e incrementar el porcentaje de personas de entre 30 y 34 años con estudios superiores completos del $31 \%$ al $40 \%$.

- Reducir el número de europeos que viven por debajo del umbral nacional de pobreza en un $25 \%$, liberando de la pobreza a 20 millones de personas.

PRIORIDADES ESTRATÉGICAS

\begin{tabular}{|c|c|c|}
\hline Crecimiento inteligente & Crecimiento sostenible & Crecimiento integrador \\
\hline $\begin{array}{l}\text { - Innovación } \\
\text { - Educación } \\
\text { - Sociedad Digital }\end{array}$ & $\begin{array}{l}\text { - Clima, energía } \\
\text { movilidad } \\
\text { - Competitividad }\end{array}$ & $\begin{array}{l}\text { - Empleo } \\
\text { cualificaciones } \\
\text { - Lucha contra la } \\
\text { pobreza }\end{array}$ \\
\hline
\end{tabular}

INICIATIVAS EMBLEMÁTICAS

\begin{tabular}{|c|c|c|}
\hline $\begin{array}{l}\text { - Unión por r la } \\
\text { innovación } \\
\text { - Juventud } \\
\text { movimiento } \\
\text { - Una agenda } \\
\text { para Europa }\end{array}$ & $\begin{array}{l}\text { - Una Europa que utilice } \\
\text { eficazmente los recursos } \\
\text { - Una política industrial } \\
\text { para la era de la } \\
\text { mundialización }\end{array}$ & $\begin{array}{lr}\text { - Agenda de } & \text { nuevas } \\
\text { cualificaciones } & y \\
\text { empleos } & \\
\text { - Plataforma europea } \\
\text { contra la pobreza }\end{array}$ \\
\hline
\end{tabular}

\section{Tabla 1: Prioridades estratégicas (Comisión Europea 2010, 36).}

Los objetivos que estableció la iniciativa europea para acompañar estas prioridades, siguieron la pauta inaugurada por la Comisión Delors, en 1993, centrada en un modelo social activo, de creación de empleo, reducción de la pobreza, disminución del abandono escolar e inversión en recursos humanos (Barbier 2012, 247). En la misma línea, la Comisión había planteado ya, para el primer decenio del nuevo siglo, hacer de Europa la economía basada en el conocimiento más dinámica y competitiva del mundo, con un sostenido crecimiento económico y una duradera cohesión social (Pérez 2004). 
Estas prioridades y sus líneas estratégicas dieron pie a siete iniciativas emblemáticas; ${ }^{1}$ en este trabajo nos centraremos en la tercera, Una agenda digital para Europa.

Lo primero que llama la atención es la precisión -y restricción- del objetivo de la iniciativa: "acelerar la implantación de internet de alta velocidad y beneficiarse de un mercado único digital para familias y empresas" (Comisión Europea 2010, 36). Parece claro que el alcance de esta agenda nace sujeto a los beneficios económicos y sociales que pudiera alcanzar ayudado por sus metas intermedias: un marco jurídico para estimular las inversiones en infraestructura de internet de alta velocidad, una política eficaz relativa al espectro, la facilitación del uso de los fondos estructurales de la UE para conseguir lo anterior y un mercado único de contenido y servicios en línea para apoyar la conformación de una gobernanza mundial de internet. Así mismo, la Comisión se proponía trabajar con el fin de reformar los fondos destinados a la investigación para incrementar el apoyo al desarrollo de las TIC, reforzar la solidez tecnológica y promover la alfabetización digital (Comisión Europea 2010, 17-18).

La orientación de la Comisión no es fortuita, sino que continúa la dirección instaurada, por lo menos, en las dos décadas anteriores. En la evolución reciente de la Comisión Europea, el período de 1995 a 2004 estuvo marcado por una actividad intensa y un particular protagonismo de los actores sociales, a quienes era atribuida una notable pertinencia económica. Para el siguiente período, de 2005 a 2009, el equilibrio entre la Comisión y los Estados miembro se fue desplazando hacia estos últimos. Al mismo tiempo, bajo la presidencia Barroso, la Comisión redujo la actividad social y se inclinó a favor de los actores económicos (Barbier 2012).

Europa 2020 tiene su antecedente en diversos programas y estrategias de la década del 2000 como eEurope en los años 2002 y 2005, o i2010, proyectado para el fortalecimiento de una sociedad del conocimiento europea sobre los principios de la estrategia de Lisboa (Sebastián et al. 2014). Victoria Camps (2004), invitada a un proyecto sobre la promoción de la alfabetización digital financiado por el programa eLearning de la Comisión Europea (2004-2006) ${ }^{2}$,

\footnotetext{
${ }^{1}$ Estas son, de acuerdo con la prioridad a que responden: (1) Crecimiento inteligente, que comprende (1.1) Unión por la innovación (innovación), (1.2) Juventud en movimiento (educación) y (1.3) Una agenda digital para Europa (sociedad digital). (2) Crecimiento sostenible, dividido en (2.1) Una Europa que utilice eficazmente los recursos (clima, energía y movilidad) y (2.2) Una política industrial para la era de la mundialización (competitividad). Finalmente, (3) Crecimiento integrador, que incluye (3.1) Agenda de nuevas cualificaciones y empleos, y (3.2) Plataforma europea contra la pobreza (Comisión Europea 2010, 36).

${ }_{2}^{2}$ Véase la decisión 2318/2003/CE del Parlamento Europeo y del Consejo, del 5 de diciembre de
} 
advertía ya sobre el reduccionismo tecnológico de esta tendencia y la necesidad, en cambio, de aprender otros lenguajes y experimentar nuevas formas de ver el mundo y relacionarse con las cosas.

En la década actual, la Comisión continúa favoreciendo los objetivos macroeconómicos y financieros, proponiéndose explotar mejor el potencial de las tecnologías de la información a favor de la innovación, el crecimiento económico y el progreso, mientras que su política social cuenta con una financiación exigua, una legislación exigente y un discurso profuso que incrementan la incertidumbre (Barbier 2012, 417).

Lo que está claro es que más que relegar la política social, lo que ha hecho la Comisión es transformarla y hacerla descansar en la agenda tecnológica. En ella, las políticas europeas apuestan cada vez con mayor fuerza por la Sociedad de la Información y el Conocimiento como clave para potenciar el crecimiento económico, la competitividad y el empleo (Sebastián et al. 2014, 105), en detrimento, no obstante, de una perspectiva sistémica coherente. Europa ha establecido, en definitiva, un modelo de civilización basado en el desarrollo e intercambio económico digital.

En este sentido, Europa 2020 obvia las diferencias de acceso, uso y competencia digital, eludiendo hablar de derechos fundamentales y otorgando a la educación digital un carácter meramente técnico moldeado sobre la noción de alfabetización. Por ejemplo, las dimensiones de su indicador de economía y sociedad digital DESI (Digital Economy and Society Index ${ }^{3}$ ), a saber, (i) conectividad, (ii) capital humano, (iii) uso de internet, (iv) integración de la tecnología digital y (v) servicios públicos digitales, reducen la competencia digital a marcadores instrumentales. Aun el criterio del "capital humano", se agota en la medición de las habilidades en el uso y desarrollo de las herramientas digitales, sin aludir a las condiciones materiales o educativas que suministrarían una base más firme al crecimiento tecnológico y otorgarían un sentido de civilización más profundo.

\section{La ciudadanía digital}

La ciudadanía, como es bien sabido, es la condición otorgada al individuo en cuanto miembro de una comunidad organizada. No quiero reproducir aquí la historia de la ciudadanía sino señalar su relación con el modelo social que la

2003. Diario Oficial $\mathrm{n}^{\circ}$ L 345 de 31/12/2003, pp. 0009-0016. Disponible en http://eurlex.europa.eu/legal-content/ES/TXT/?uri=celex:32003D2318 (última consulta 15/01/2016).

${ }^{3}$ Véase <http://ec.europa.eu/digital-agenda/en/digital-agenda-scoreboard> 
enmarca. En este sentido, la ciudadanía digital está comprendida en el modelo de la sociedad del conocimiento y la información (Robles 2009).

Una de las primeras cuestiones que surgen en la consideración de la ciudadanía digital es la ausencia de una comunidad organizada y las nociones inéditas de espacio digital y comunidad virtual (Robles 2009, 55), o la de comunidades virtuales "geográficamente dispersas". Esta dispersión, no obstante, es por lo menos contradictoria, debido a la paradójica relación que existe entre dispersión y presencia, o dispersión y concentración en estas comunidades caracterizadas por el acceso a internet (Vallespín 2015).

Lo anterior ha supuesto un reto a la noción convencional de ciudadanía y a las legislaciones de los países, obligadas a responder a las circunstancias de la sociedad del conocimiento y la información (Castells 1997). Sin embargo, la trayectoria misma del concepto de sociedad de la información es ambigua y contradictoria, tal como el desarrollo del capitalismo que lo ha visto nacer. Al final -tal parece- esta ha terminado por adquirir su condición de evidencia, "sin que los ciudadanos hayan podido ejercer su derecho a un verdadero debate" (Mattelart 2007, 12), convirtiéndose en el paradigma dominante del cambio y la garantía indiscutible de un mundo mejor.

La ciudadanía digital no es, en el presente, una idea original, pero su relación con una gran variedad de perspectivas, desde la ampliación de la democracia (Meneses 2015) y la reconsideración de la brecha digital (Alva 2015, Segev 2010), hasta la netiqueta o el comportamiento apropiado en entornos comunicativos virtuales y el uso responsable de la tecnología (Isman y Gungoren 2013) la refrescan. Es igualmente interesante la diversidad de problemáticas relevantes que puede abarcar, como la confección de una ética reticular y la reconsideración del paradigma de la ciudadanía digital en las sociedades multiculturales (Bustamante 2011, 2013) la formalización de los derechos humanos digitales o derechos humanos de cuarta generación (Bustamante 2010, Martínez-Villalba 2014), la vigilancia y el control masivo de la privacidad del ciudadano (Estévez 2015, Lyon 2007) o las consecuencias para la educación y el trabajo de una sociedad formada por sujetos nómadas (Moravec 2013, Cobo y Moravec 2011). Al mismo tiempo, la nueva dimensión de lo público, que sobrepasa los límites de los Estados nacionales, da un nuevo sentido a lo político y sigue representando un reto para los países en particular.

\section{Acuerdos generales}

Más allá de la diversidad de perspectivas, descubro que existe un acuerdo general en dos puntos relacionados con el objetivo primordial de este trabajo. 
El primero es el acuerdo generalizado en una serie de requisitos y derechos que los países y las organizaciones políticas internacionales deberían garantizar, cumplir y promover. Estas serían, entre otras, la extensión en el acceso y uso de internet así como en la percepción de su utilidad; la evolución de los servicios y herramientas a disposición de los ciudadanos, principalmente en relación con las actividades políticas, sociales, administrativas y/o legales; el tratamiento legal y regulado de las relaciones de la ciudadanía con sus representantes por medios virtuales $\mathrm{y}$, en general, la apropiación progresiva de internet por parte de la sociedad (Robles 2009, 66-67).

No podemos obviar que las condiciones traídas por la Sociedad de la Información y el Conocimiento han dado lugar al surgimiento de un nuevo mapa de desigualdades, como afirma Armand Mattelart al formular, basado en la noción de economía-mundo de Fernand Braudel, el concepto comunicaciónmundo (Mattelart y Multigner 1993, Mattelart 1998, 100). De hecho, la sociedad de la información y la comunicación, solo revela su verdadero sentido dentro de una configuración geopolítica (Mattelart 2007, 12).

Desigualdad y brecha digital

La era digital es, más que un mundo de innovación, conveniencia y prosperidad incesantes, un desafío que amenaza constantemente la simetría legal, política e identitaria de la ciudadanía digital (Robles 2009, 89). Cuando estas dimensiones entran en desequilibrio, el acceso y la ciudadanía se convierten en una suerte de línea abismal (Santos 2010), es decir, el privilegio de "unos" que establecen así un lado de la línea, frente a "los otros", privados de derechos, posibilidades políticas y oportunidades de identificación.

El monopolio de la violencia legítima con que Weber definió el Estado, es hoy, afirma Elad Segev (2010), el "monopolio del conocimiento y la comunicación", ya sea por una forma de comunicación dominante, una fuente y tecnología dominante o una clase o grupo dominante. En el mismo sentido, la división digital puede ser establecida global o socialmente, según la diferencia que opere en el acceso a internet y su estructura, ya sea entre países o entre grupos sociales. También puede ser una división democrática, de acuerdo con la manera en que las aplicaciones y los usos de la información online permita que el ciudadano se pueda captar, movilizar o participar en la vida pública (Norris 2001, Segev 2010, 7). 


\section{La competencia digital}

Una vez establecidos los requisitos y derechos fundamentales que, ante la afirmación de la sociedad del conocimiento los países y organizaciones conviene proteger, surge un segundo acuerdo en materia de ciudadanía digital: la educación, alfabetización y/o la competencia.

\section{¿Por qué hace falta ser competente?}

Que en la actualidad hace falta saber conocer y utilizar, es decir, ser competente en relación con las Tecnologías de la Información y la Comunicación, es evidente. No quiero decir con esto que el predominio de un ser/hacer técnico o instrumental sea incontestable ${ }^{4}$ pero la tecnología digital ha sido incorporada masivamente a dimensiones fundamentales de la vida social, como la educación y el trabajo, lo que hace ineludible afrontar la capacitación y educación en materia de tecnología de la información y la comunicación como una tarea primordial. En el papel, la alfabetización y educación digital tendría que ser tan avanzada como la tecnología de la información y la comunicación, pero es, en cambio, pasiva y acrítica (Van Dijk 2004).

Por otro lado, la omnipresencia digital acentúa la importancia de redefinir la alfabetización y el "aprendizaje competente", al igual que las metodologías o estrategias de enseñanza más acordes con las competencias informacionales y digitales de nuestros tiempos (Area y Guarro 2012).

La digitalización de la información está permitiendo crear nuevas formas de codificación, representación y construcción de la cultura en las que las experiencias analógicas a menudo se confunden con las virtuales, propiciando un ida y vuelta entre estos dos escenarios de interacción sociocultural, y evidenciando que, en las últimas décadas, la influencia de los medios de comunicación no ha ido a la par del fomento de la alfabetización mediática (García-Ruiz et al. 2014).

En España, la Ley General de la Comunicación Audiovisual del 1 de abril de 2010 respondió a la necesidad de alfabetización mediática de los ciudadanos, poniendo especial atención en la formación de prosumidores, es decir, aquellos usuarios de la Red que asumen el rol de canal de comunicación y no solo

\footnotetext{
${ }^{4}$ Véanse, en este sentido, los trabajos críticos de Jacques Ellul, Lewis Munford, Günther Anders, Proudhon o Illich.
} 
consumen contenidos de información, sino que los producen (Ministerio de Educación Cultura y Deporte 2011, Sánchez y Contreras 2012). ${ }^{5}$

\section{¿Qué nivel de competencia mediática tienen los españoles?}

No obstante, los datos sobre alfabetización mediática y digital en este país son diversos y en ocasiones discordantes. Los informes suelen dar panoramas numéricos que disimulan los desafíos más apremiantes con comparaciones celebratorias (Fundación Telefónica 2015). Algunos otros incluyen estudios más mesurados, pero desde una perspectiva poco incisiva (Moreno 2015). Los más críticos encuentran "carencias importantes" en la mayor parte de las dimensiones tradicionales de la competencia mediática (lenguajes, ideología y valores, producción y difusión, procesos de recepción e interacción, dimensión estética), y aunque reconocen datos más aceptables en el manejo de la tecnología en sí, destacan la baja comprensión de los procesos mentales que se producen en la interacción con las pantallas (Ferrés et al. 2012). En general, la impresión de estos autores es que la alfabetización multimedia y digital se ha centrado en incorporar las herramientas a la práctica docente -o laboral- sin una educación en medios adecuada, provocando la inmersión de los jóvenes y los trabajadores en el flujo del discurso digital sin una seria conciencia de su naturaleza y/o implicaciones. Es decir, la distancia entre el uso de los medios digitales y un conocimiento real de la capacidad y poder de los elementos visuales que los conforman es relevante.

\section{Alfabetización y/o competencia digital}

El alfabeto de las formas culturales de nuestro siglo, una sociedad multimodal e informacional, es complejo; la información, los códigos, los lenguajes y la diversidad de soportes de almacenamiento, distribución y acceso, hacen necesaria la redefinición del concepto tradicional de alfabetización para que los niños, jóvenes y adultos "puedan realizar un uso culto, crítico e inteligente de la información que se vehicula a través de las múltiples herramientas y redes de naturaleza digital" (Area y Guarro 2012, 50). Para esto, la alfabetización tendría

\footnotetext{
${ }^{5}$ La educación en competencia digital en España, está pautada por la integración de la alfabetización en medios $y$ digital de la (http://www.ite.educacion.es/formacion/materiales/8/cd_2013/m4_4/la_alfabetizacin_informacional alfin.html). Una noción interesante del documento del Ministerio de Educación para la apropiación de la alfabetización digital en las bibliotecas es el concepto de "bibliotecas híbridas" $(2011,10)$.
} 
que alcanzar distintas modalidades: en información, digital, tecnológica, alfabetizaciones múltiples, educación en medios y educación mediática.

En el marco europeo (Parlamento Europeo 2006, anexo), la competencia digital "entraña el uso seguro y crítico de las tecnologías de la sociedad de la información (TSI) para el trabajo, el ocio y la comunicación" basado en "el uso de ordenadores para obtener, evaluar, almacenar, producir, presentar e intercambiar información, y comunicarse y participar en redes de colaboración a través de Internet". 6

En teoría, "la competencia digital exige una buena comprensión y amplios conocimientos sobre la naturaleza, la función y las oportunidades de las TSI en situaciones cotidianas de la vida privada, social y profesional"; asimismo, se afirma, "las personas deben comprender las posibilidades que las TSI ofrecen como herramienta de apoyo a la creatividad y la innovación, y estar al corriente de las cuestiones relacionadas con la validez y la fiabilidad de la información disponible y de los principios legales y éticos por los que debe regirse el uso interactivo de las TSI"

De estos lineamientos se desprende un marco para desarrollar y comprender la competencia digital en Europa organizado en cinco áreas: (i) información, (ii) comunicación, (iii) creación de contenido, (iv) seguridad y (v) resolución de problemas (Ferrari 2013).

\section{Áreas de competencia}

Información

Comunicación

Creación de contenido

\section{Descripción}

Identificar, localizar, recuperar, almacenar, organizar y analizar la información digital, evaluando su finalidad y relevancia.

Comunicar en entornos digitales, compartir recursos a través de herramientas en línea, conectar y colaborar con otros a través de herramientas digitales, interactuar y participar en comunidades y redes; conciencia intercultural.

Crear y editar contenidos nuevos (desde textos a vídeos), integrar y reelaborar conocimientos $\mathrm{y}$ contenidos previos, realizar producciones artísticas, contenidos multimedia y programación informática, saber aplicar los derechos de propiedad intelectual y las licencias de uso.

\footnotetext{
${ }^{6}$ Diario Oficial de la Unión Europea, 30/12/2006, p. L 394/15.

${ }^{7}$ Diario Oficial de la Unión Europea, 30/12/2006, p. L 394/16.
} 
\begin{tabular}{ll} 
Seguridad & $\begin{array}{l}\text { Protección personal, protección de datos, protección } \\
\text { de la identidad digital, uso de seguridad, uso seguro } \\
\text { y sostenible. }\end{array}$ \\
\hline Resolución de problemas & $\begin{array}{l}\text { Identificar necesidades y recursos digitales, tomar } \\
\text { decisiones a la hora de elegir la herramienta digital } \\
\text { apropiada, acorde a la finalidad o necesidad, } \\
\text { resolver problemas conceptuales a través de medios } \\
\text { digitales, resolver problemas técnicos, uso creativo } \\
\text { de la tecnología, actualizar la competencia propia y } \\
\text { la de otros. }\end{array}$
\end{tabular}

\section{Tabla 2: Áreas de competencia del proyecto DIGCOM (Ferrari 2013).}

Para salvar la distancia entre realidad y educación digital, la alfabetización debe contemplar los nuevos códigos y las nuevas formas comunicativas de la cultura digital. En este sentido, las competencias sobre las que debe formarse el prosumidor van más allá de la adquisición de destrezas para el dominio instrumental y alcanzan la adquisición de competencias relacionadas con la búsqueda, el análisis, la selección y la comunicación de datos (Sánchez y Contreras 2012). Este nuevo usuario -no solamente usuario-, es capaz de dinamizar la comunicación e interacción entre emisores y receptores, organizar los recursos requeridos para la producción de contenidos creativos, críticos y responsables, y producir y realizar nuevos mensajes con suficiente calidad tecnológica, artística, ética y moral (García-Ruiz et al. 2014, 17). Podríamos pensar también en la competencia de apropiarse y otorgar significado a la información disponible en la Red y sus distintos lenguajes expresivos (AreaMoreira y Ribeiro-Pessoa 2012, 17).

No obstante, aunque es clara la relación entre la formación como prosumidor y el empoderamiento de los ciudadanos, en la práctica estos distan de ser las áreas prominentes de la alfabetización digital. Existe, por el contrario, una baja participación (Sánchez y Contreras 2012), o "participación creciente" (Ferrés et al. 2012) en los entornos digitales dirigidos a la educación, producto del vacío educativo de los medios de comunicación en la era digital o, por lo menos, de la desigual relación entre la influencia de los medios de comunicación y el fomento de la alfabetización mediática (García-Ruiz et al. 2014).

Cobran sentido, en este contexto, la pedagogía de la multialfabetización (Cope y Kalantzis 2010) o nuevas alfabetizaciones (Lankshear y Knobel 2009) que recogen la tradición liberadora y dialógica de Paulo Freire, el enfoque de la formación democrática del ciudadano de John Dewey y la educación mediática crítica, además de reconocer la heterogeneidad de la sociedad y adaptarse a su 
condición de ciudadano y trabajador (Area y Guarro 2012). Otra imagen de la plasticidad que requiere la nueva educación digital la podemos encontrar en las nociones de knowmad society (Moravec 2013) y aprendizaje invisible (Cobo y Moravec 2011) que presentan un arquetipo sociotecnológico educativo que considera el aprendizaje como un continuum multimodal y que actualiza la discusión sobre los alcances de la educación fuera de la educación formal. ${ }^{8}$

\section{Propuestas}

Siguiendo la propuesta de Area y Pessoa (2012), además de los componentes instrumental, cognitivo-intelectual y sociocomunicacional, la alfabetización en medios digitales tendría que incorporar de una manera más firme, las competencias axiológica y emocional.

La primera está relacionada con la toma de conciencia de que las TIC no son asépticas ni neutrales desde el punto de vista social, sino que inciden en el entorno cultural y político con su propia carga de valores éticos y democráticos; la segunda se relacionaría con los afectos, sentimientos y pulsaciones emocionales causadas por la experiencia en entornos virtuales.

Podríamos agrupar los alicientes para la alfabetización y educación digital en dos conjuntos; por un lado, los intereses para la formación del prosumidor, y por otro, los aspectos clave para el ejercicio de la ciudadanía. En el primer grupo nos podríamos ceñir a tres habilidades básicas: dinamizar, organizar y realizar o producir, mientras que en el segundo podríamos considerar tres o cuatro aspectos clave: educar, empoderar y proteger, ${ }^{9}$ o respetar, educar y proteger (Isman y Gungoren 2013). En el mismo sentido, Isman y Gungoren (2014), presentan otras características que debería tener la ciudadanía digital: entendimiento humano, la inclusión de temas sociales y culturales relacionados con la tecnología y la práctica legal, el comportamiento ético, la defensa y práctica de la seguridad, el uso responsable y legal de la información y la tecnología, una actitud positiva hacia el uso de la tecnología que comprende colaboración, aprendizaje y productividad, la exhibición de responsabilidad personal para el aprendizaje continuo y el liderazgo.

Por último, como una arista de la ciudadanía global, la ciudadanía digital debe ser considerada un requisito para la diversidad y la coexistencia creativa (Ikeda 2013) en una cultura de participación (Grizzle 2014), aunque más que

\footnotetext{
${ }^{8}$ Illich, Freire, Argyris o Knowles pueden ser ejemplos de este entendido.

${ }^{9}$ Esta es la propuesta de Common Sense Media, organización sin ánimo de lucro dedicada a la educación y la promoción de la seguridad en el uso de la tecnología y los medios de comunicación masiva. Véase https://www.commonsensemedia.org/educators
} 
como una condición, como una tendencia o aspiración (Searson et al. 2015). De acuerdo con Parekh $(2002,2005)$, más que una ciudadanía digital absoluta, los ciudadanos deberían ser orientados globalmente y capaces de llevar a cabo sus responsabilidades como ciudadanos democráticos sin cargar con ellas a los "otros globales", priorizando el interés de la humanidad sobre la gobernanza homogeneizadora. Así, la ciudadanía globalmente orientada, debería estar en constante examen de sus propias políticas, mantener un interés activo en los asuntos de otros países y el compromiso de crear un orden mundial más justo (Searson et al. 2015). En el mismo sentido, la alfabetización debe ser entendida como un permitidor (enabler) y no como un constructo cultural.

Ahora bien, aunque las nuevas tecnologías obligan a las organizaciones sociales a articular redes alternativas de cooperación y acción colectiva, transformando el capital social en capital social interconectado, no hay que cerrar los ojos a que hay una "desigual conformación del espacio de integración económica" en nuestro estadio de "capitalismo cognitivo" (Caballero 2012, Zafra 2015). Si en el mundo digital, como dijo McLuhan, "el medio es el mensaje", en el medio digital "el mensaje es todo ciudadano", y la atención de los media y la tecnología se traslada de la tecnología hacia los individuos, las comunidades y sus interacciones con la información y el conocimiento (Grizzle 2014).

\section{Bibliografía}

Alva de la Selva, Alma Rosa. "Los nuevos rostros de la desigualdad en el siglo XXI: la brecha digital." Revista Mexicana de Ciencias Políticas y Sociales 60, no. 223 (Enero 2015): 265-85. doi:10.1016/S0185-1918(15)72138-0.

Area-Moreira, Manuel, y Maria Teresa Ribeiro-Pessoa. "De lo sólido a lo líquido: las nuevas alfabetizaciones ante los cambios culturales de la Web 2.0." Comunicar 19, no. 38 (Marzo 2012): 13-20. doi:10.3916/C38-2012-02-01.

Area, Manuel, y Amador Guarro. "La alfabetización informacional y digital: fundamentos pedagógicos para la enseñanza y el aprendizaje competente." Revista Española de Documentación Científica, No. Monográfico (2012): 46-74. Barbier, Jean-Claude. "El destino de la política social en la UE. Evolución del discurso político de la estrategia de Lisboa a Europa 2020." Revista Internacional del Trabajo 131, no. 4 (Diciembre 2012): 415-41. doi:10.1111/j.1564-9148.2012.00155.x.

Bustamante Donas, Javier. "Development of a Netlike (Reticular) Ethics as a Paradigm for Digital Citizenship in a Multi-Cultural Society." Nomads. Critical Review of Social and Juridical Sciences 1, no. 2 (2011): 81-90. 
"Ética en la nube: dilemas éticos y políticos en el modelo de computación en nube (Cloud Computing)." Argumentos de Razón Técnica: Revista Española de Ciencia, Tecnología y Sociedad, y Filosofía de la Tecnología, no. 16 (2013): 37-54.

—. "La cuarta generación de Derechos Humanos en las redes digitales: segundos pensamientos." Telos, no. 85 (2010): 80-89.

Caballero, Francisco Sierra. "Ciudadanía digital y sociedad de la información en la Unión Europea. Un análisis crítico." Andamios. Revista de Investigación Social 9, no. 19 (2012): 259-82.

Camps, Victoria. "Fundamentación intelectual y social de la Digital Literacy." En Promoting Digital Literacy. Informe Final EAC/76/03, 46-52. Barcelona: Gabinete de Comunicación y Educación - Universidad Autónoma de Barcelona, 2004.

Caridad Sebastián, Mercedes, Ana María Morales García y Fátima García López. "La estrategia Europa 2020 y la sociedad de la información como instrumentos de cohesión e integración en época de crisis: ¿Utopía o realidad?” Investigación Bibliotecológica 28, no. 64 (2014): 101-15.

Castells, Manuel. La Era de La Información: Economía, Sociedad y Cultura. Madrid : Alianza, 1997.

Cobo Romaní, Cristóbal, y John W. Moravec. Aprendizaje Invisible. Hacia Una Nueva Ecología de La Educación. Barcelona: Laboratori de Mitjans Interactius Publicacions i Edicions de la Universitat de Barcelona, 2011.

Comisión Europea. "Europa 2020. Una estrategia para un crecimiento inteligente, sostenible e integrador." Revista del Ministerio de Trabajo e Inmigración. Bruselas: Subdirección General de Publicaciones, 2010.

Cope, Bill, y Mary Kalantzis. “Multialfabetización': nuevas alfabetizaciones, nuevas formas de aprendizaje." Boletín de la Asociación Andaluza de Bibliotecarios. Asociación Andaluza de Bibliotecarios, AAB, 2010.

Estévez Araujo, José A. (Ed.). La democracia en bancarrota. Madrid: Trotaa, 2015.

—. "La Sociedad de La Vigilancia." Mientrastanto.e 141, no. Diciembre (2015). http://www.mientrastanto.org/boletin-141/ensayo/la-sociedad-de-lavigilancia.

Ferrari, Anusca. "DIGCOMP: A Framework for Developing and Understanding Digital Competence in Europe." Sevilla, 2013.

Ferrés Prats, Joan, Ignacio Aguaded-Gómez y Agustín García-Matilla. "La competencia mediática de la ciudadanía española: dificultades y retos." Revista ICONO14. Revista científica de Comunicación y Tecnologías emergentes 10, no. 3 (Octubre 2012): 23-42. doi:10.7195/ri14.v10i3.201. 
Fundación Telefónica. La Sociedad de la Información en España 2014. Madrid: Ariel - Fundación Telefónica, 2015.

García-Ruiz, Rosa, Antonia Ramírez-García y María del Mar Rodríguez-Rosell. "Educación en alfabetización mediática para una nueva ciudadanía prosumidora." Comunicar 22, no. 43 (July 1, 2014): 15-23. doi:10.3916/C432014-01.

Grizzle, Alton. "MIL, Intercultural Dialogue and Global Citizenship." In Global Citizenship in a Digital World. MILID Yearbook 2014. Sherry Hope Cullver y Paulette A. Kerr (Eds.), 17-26. Gotemburgo, SE: Nordicom, 2014.

Ikeda, Daisaku. Amor compasivo, sabiduría y valentía: por una sociedad global de Paz y de coexistencia creativa. Rivas: Ediciones Civilización Global, 2013. Isman, Aytekin y Ozlem Canan Gungoren. "Being Digital Citizen." Procedia Social and Behavioral Sciences 106 (December 2013): 551-56. doi:10.1016/j.sbspro.2013.12.063.

"Digital Citizenship." TOJET: The Turkish Online Journal of Educational Technology 13, no. 1 (2014): 73-77.

Lankshear, Colin y Michele Knobel. Nuevos Alfabetismos. Su práctica cotidiana y el aprendizaje en el aula. Madrid: Morata, 2009.

Lyon, David. Surveillance Studies: An Overview. Cambridge: Polity, 2007.

Martínez-Villalba, Juan Carlos Riofrío. "La cuarta ola de derechos humanos: los derechos digitales." Revista Latinoamericana de Derechos Humanos, Marzo 2014.

Mattelart, Armand. Historia de la sociedad de la información. Barcelona: Paidós, 2007.

—. La mundialización de la comunicación. Barcelona: Paidós, 1998.

Mattelart, Armand y Gilles Multigner. La Comunicación-Mundo: historia de las ideas y de las estrategias. Madrid: Fundesco, 1993.

Meneses Rocha, María Elena. Ciberutopías. Democracia, redes sociales y movimientos-red. México: Porrúa-ITESM, 2015.

Ministerio de Educación Cultura y Deporte. Marco de Referencia Para Las Bibliotecas Escolares. Madrid: Secretaría General Técnica - Subdirección General de Documentación y Publicaciones, 2011.

Moravec, John W. Knowmad Society. Education Futures, 2013.

Moreno, María Dolores. "La sociedad española y la alfabetización multimedia: internet, redes sociales y competencia tecnológica." Valencia, 2015.

Norris, Pippa. Digital Divide: Civic Engagement, Information Poverty, and the Internet Worldwide. Cambridge: Cambridge University Press, 2001.

Parekh, Bhikhu C. "Cosmopolitanism and Global Citizenship." Review of International Studies 29, no. 1 (Diciembre 2002): 3-17. 
doi:10.1017/S0260210503000019.

. Repensando el multiculturalismo: diversidad cultural y teoría política. Madrid: Istmo, 2005.

Parlamento Europeo. Recomendación del Parlamento Europeo y del Consejo del 18 de Diciembre de 2006 sobre las competencias clave para el aprendizaje permanente (2006/962/CE), 2006.

Pérez Tornero, José Manuel. "Promoting Digital Literacy. Informe Final EAC/76/03." Barcelona, 2004.

Robles Morales, José Manuel. Ciudadanía digital : una introducción a un nuevo concepto de ciudadano. Barcelona: Editorial UOC, 2009.

Sánchez Carrero, Jacqueline y Paloma Contreras Pulido. "De cara al prosumidor: producción y consumo empoderando a la ciudadanía 3.0." Revista ICONO14. Revista científica de Comunicación y Tecnologías emergentes 10, no. 3 (Octubre 2012): 62-84. doi:10.7195/ri14.v10i3.210.

Santos, Boaventura de Sousa. Para descolonizar Occidente: más allá del pensamiento abismal. Buenos Aires: CLACSO-Prometeo Libros, 2010.

Searson, Michael, Marsali Hancock, Nusrat Soheil y Gregory Shepherd. "Digital Citizenship Within Global Contexts." Education and Information Technologies 20, no. 4 (2015): 729-41.

Segev, Elad. Google and the Digital Divide. The Bias of Online Knowledge. Oxford: Chandos Publishing, 2010.

Vallespín Oña, Fernando. "La gestión de la democracia en el entorno digital. Política y Nuevas Redes." Telos, no. 100 (2015): 45-48.

Van Dijk, Teun. "Digital Literacy and Critical Discourses Studies." In Promoting Digital Literacy. Informe Final EAC/76/03, 64-72. Barcelona: Gabinete de Comunicación y Educación - Universidad Autónoma de Barcelona, 2004.

Zafra Díaz, Juan, and Juan M Zafra Díaz. "Sociedad Digital. Participación y Nuevos Liderazgos.” Telos, no. 100 (2015): 55-57. 
